\title{
Prognostic significance of altered miRNA expression in whole blood of OSCC patients
}

\author{
JUTTA RIES $^{1}$, CHRISTOPH BARAN ${ }^{1}$, FALK WEHRHAN $^{1}$, MANUEL WEBER ${ }^{1}$, FRIEDRICH W. NEUKAM ${ }^{1}$, \\ ANDREA KRAUTHEIM-ZENK ${ }^{1}$ and EMEKA NKENKE ${ }^{2}$ \\ ${ }^{1}$ Department of Oral and Maxillofacial Surgery, Erlangen University Hospital, D-91054 Erlangen, Germany; \\ ${ }^{2}$ Department of Oral and Maxillofacial Surgery, Medical University Vienna, A-1090 Vienna, Austria
}

Received October 6, 2016; Accepted November 23, 2016

DOI: $10.3892 /$ or.2017.5639

\begin{abstract}
Currently, there is a lack of blood markers for the detection of recurrent oral squamous cell carcinoma (OSCC). The present study aimed to investigate whether the aberrant expression of single microRNAs (miRNAs) in whole blood of patients could serve as a biomarker for persistent or recurrent OSCC. Whole blood of 2 groups of formerly treated OSCC patients was investigated by RT-qPCR for their circulating miRNA profiles. The R-OC group included patients with recurrence of OSCC $(n=21)$ and the NR-OC group included patients without recurrence $(n=21)$. Fold-changes and significance of the differences in miRNA expression levels between the groups were determined. A cut-off point (COP) for the discrimination between the R-OC and NR-OC groups was calculated and the significance between over/under expression of the miRNAs and the recurrence of malignancy was determined. Significant differences in the miRNA expression in whole blood of the R-OC and NR-OC groups were found. The levels of miR-3651 and miR-494 were significantly increased and the level of miR-186 was significantly decreased in whole blood of the R-OC patients $\left(\mathrm{p}_{\mathrm{miR}-3651}=0.001, \mathrm{p}_{\mathrm{miR}-494}=0.003\right.$ and $\left.\mathrm{p}_{\mathrm{miR}-186}=0.001\right)$. By the determination of the COP, increased or decreased expression of the markers was significantly correlated to the recurrence of the disease. Altered expression of miR-494, miR-3651 and miR-186 appears to be associated with the recurrence of OSCC. The present study may form the basis for establishing a blood test as a minimally invasive method for the detection of the recurrence of OSCC.
\end{abstract}

\section{Introduction}

Late diagnosis, frequent regional lymph node metastases and the high rate of local and regional recurrence that is caused

Correspondence to: Dr Jutta Ries, Department of Oral and Maxillofacial Surgery, University Hospital Erlangen, Glückstrasse 11, D-91054 Erlangen, Germany

E-mail: Jutta.Ries@uk-erlangen.de

Key words: OSCC, recurrence, whole peripheral blood, miRNA expression, RT-qPCR mainly by the persistence of malignant cells, are the major causes for the poor prognosis of oral squamous cell carcinoma (OSCC) $(1,2)$. Recurrence leads to a significantly decreased life expectancy of the affected patients (3). Early detection of recurrence improves patient survival (4-7).

TNM classification, histological grade and the depth of tumor invasion are the best known prognostic factors for recurrence, even if the real prognostic value of these clinical and pathological features is still controversial $(2,8)$. Additionally, the state of the margins after tumor resection has been investigated in order to predict recurrence. However, even following wide tumor excision, a local recurrence of OSCC occurs in up to $30 \%$ of OSCC patients due to residual malignant cells that could not be microscopically detected in tumor margins at the time of surgery (9).

To solve these shortcomings of the clinical parameters, there is an urgent need to determine highly sensitive and tumor-specific biomarkers for the stratification of patients with high risk of disease recurrence and for long-term surveillance of OSCC as well as for restaging procedures to exclude or confirm tumor recurrence at stages that allow a successful therapeutic intervention (10). Unfortunately, intensive research spanning several decades has failed to identify new prognostic molecular markers in oral cancer. To date, relevant prognostic indicators with sufficient sensitivity and specificity are nonexistent (11). Therefore, the importance of developing useful diagnostic and monitoring tools is emphasized in order to improve the clinical outcome of patients suffering from OSCC.

MicroRNAs (miRNAs) are predicted to control $\sim 50 \%$ of all gene expression (12). Thus, altered miRNA expression has been associated with several diseases including the development of different types of cancers $(13,14)$. In addition, miRNAs are released from cells and enter the circulation, where they are highly stable. They have been found in several human body fluids, including plasma, serum, whole blood cells and saliva (15). The alterations in cancer cells are thought to be reflected in the extracellular space as affected cells release upregulated miRNAs, fail to release apparently downregulated species or export selectively the type of miRNAs which suppress tumorigenesis.

Therefore, the detection of altered patterns of circulating miRNAs in the blood has been proposed as cancer biomarkers used in minimally invasive assays that may aid in risk 
Table I. Demographic characteristics of the test (OSCC patients), control (healthy volunteers) and follow-up groups.

\begin{tabular}{lcccc}
\hline Group & Test group & Control group & R-OC group & NR-OC group \\
\hline Number of cases & 54 & 33 & 21 & 21 \\
Mean age \pm SD (years) & $65.04 \pm 10.97$ & $60.5 \pm 20.68$ & $60.43 \pm 8.25$ & $62.1 \pm 12.96$ \\
Age range (years) & $35-93$ & $15-88$ & $51-83$ & $33-81$ \\
Gender, n (\%) & & & & \\
Male & $37(68.5)$ & $23(69.7)$ & $16(76.2)$ & $14(66.7)$ \\
Female & $17(31.5)$ & $10(30.3)$ & $5(23.8)$ & $7(33.3)$ \\
\hline
\end{tabular}

R-OC, tumor patients suffering from a recurrence within 1 year; NR-OC, patients with no recurrence within 1 year. SD, standard deviation.

assessment, diagnosis, prognosis, detection of recurrence, monitoring of the clinical course of the disease, and in predicting or monitoring treatment response as well as in therapy decision making in various types of cancer $(11,16-24)$ including OSCC (25-27). Ultimately, it has been proven that alterations in the patterns of miRNAs predict recurrence risk and are associated with therapeutic response (28-30). Recently, we demonstrated that the miRNAs, miR-494, miR-3651 and miR-186, are differentially expressed in whole blood of OSCC patients when compared to that of healthy volunteers. The altered expression rates were significantly correlated to diagnosis. Additionally, overexpression of miR-3651 was associated to lymph node metastases, clinical stage and to more dedifferentiated tumors. Hence, these miRNAs may be useful in diagnostic and prognostic applications $(31,32)$. Nevertheless, little is known concerning altered miRNA expression in whole blood of recurrent OSCC patients (33-36).

The present study aimed to evaluate the difference in circulating miRNA expression in whole blood samples of OSCC patients suffering from recurrence compared to patients who were disease-free for at least 1 year after treatment in order to clarify their impact in the early detection of recurrent disease.

\section{Materials and methods}

Patients and sample collection. The present study was approved by the Ethics Committee of the University of Erlangen-Nuremberg (Erlangen, Germany) (approval no. 3962) and patient written informed consent was obtained.

Patients who suffered from a primary OSCC in the past were included. They were clinically and histopathologically considered to be free of tumor cells after surgical removal of the tumor. The patients were followed up for 1 year at time-points of 1, 3, 6, 9 and 12 months after primary treatment in order to detect recurrence of disease. Based on these observations 2 groups were established. The R-OC group included 21 OSCC patients who suffered from recurrence within 1 year. Blood samples were immediately obtained after clinical observation of the occurrence of recurrent disease. The 21 patients of the NR-OC group were free of disease for at least 1 year after primary treatment. Fifty-four patients with an initial diagnosis of OSCC and 33 healthy volunteers served as control groups. Demographic information concerning the tumor, control, R-OC and NR-OC cohorts is presented in Table I.
Sampling of whole blood and miRNA isolation. Two aliquots of whole blood $(2.5 \mathrm{ml})$ for each subject (tumor, R-OC, NR-OC and control group) were collected in a PAXgene Blood RNA Tube (PreAnalyticX, Hombrechtikon, Switzerland) and stored at $-80^{\circ} \mathrm{C}$ until RNA isolation.

Whole RNA was extracted using the PAXgene Blood miRNA kit as recommended by the manufacturer. The RNA concentration was assessed using NanoDrop spectrometer (PeqLab, Erlangen, Germany), and sufficient quality of the samples for RT-qPCR analysis was confirmed using A260/A280 ratios. Subsequently the RNA samples were stored at $-80^{\circ} \mathrm{C}$.

Real-time quantitative reverse transcription-PCR ( $R T-q P C R)$. The values of the miRNAs, miR-186, miR-494 and miR-3651, were determined by RT-qPCR. These analyses were carried out on $500 \mathrm{ng}$ of total RNA. In the first step, miRNA was reverse transcribed using the miScript II RT kit according to the manufacturer's recommendations (Qiagen, Hilden, Germany). Detection of amplification was carried out on $2.5 \mathrm{ng}$ of cDNA using the miScript SYBR-Green PCR kit and miRNA-specific quantitative RT-PCR primer sets for the miRNA of interest (Qiagen) were determined on an ABI-7300 Sequence Detection System (Applied Biosystems, Foster City, USA). The features of the miRNAs are summarized in Table II.

The values from the RT-qPCR analyses were normalized by the $\triangle \mathrm{CT}$ method using the primer sets RNU6-2 (U6 snRNA, RNA U6 small nuclear 2) and SNORD44 (small nucleolar RNA, C/D box 44) as internal controls (Qiagen; Table II). The mean value of both controls was applied as the normalization value.

Relative quantification of differences in expression (RQ) between the 2 groups was carried out by the $\Delta \Delta \mathrm{CT}$ method using the formula ( $R Q=2^{-\Delta \Delta C T}$ ) (37). A 2-fold change in miRNA expression rate $(0.5 \leq \mathrm{RQ} \leq 2)$ between the 2 groups was defined as relevant.

Statistical analysis. For statistical evaluation, IBM ${ }^{\circledR}$ SPSS Statistics 21 (SPSS, Inc., Chicago, IL, USA) was used. The mean value of duplicate $\triangle \mathrm{CT}$ values for each sample was used for the data results. Expression data were controlled for normal distribution using the Shapiro-Wilk test. Graphical diagrams are plotted as Box-Whisker plots which represent the median (ME), the interquartile range (IQR) and the standard deviation (SD) as well as the minimum and 
Table II. List of miRNAs, snRNAs (endogenous controls) and miScript Primer Assay used in the RT-qPCR analysis.

\begin{tabular}{llll}
\hline Sanger ID & $\begin{array}{c}\text { Sanger accession } \\
\text { Mature miRNA }\end{array}$ & Sequence & $\begin{array}{c}\text { Ref. no. } \\
\text { (Qiagen) }\end{array}$ \\
\hline $\begin{array}{l}\text { miRNAs } \\
\text { hsa-miR-186-5p }\end{array}$ & MIMAT0000456 & CAAAGAAUUCUCCUUUUGGGCU & MS 00008883 \\
hsa-miR-3651 & MIMAT0018071 & CAUAGCCCGGUCGCUGGUACAUGA & MS 00023121 \\
hsa-miR-494-5p a $^{\text {a }}$ & MIMAT0026607 & AGGUUGUCCGUGUUGUCUUCUC & MSC 0002535 \\
Endogenous controls & Ref. Seq & & \\
RNU6-2 & NR_002752 & & MS 00033740 \\
SNORD44 & NR_002750 & MS 00007518 \\
\hline
\end{tabular}

${ }^{\mathrm{a} C u s t o m ~ m i S c r i p t ~ P r i m e r ~ A s s a y . ~}$

maximum values of the $\Delta C T$ values. Statistical relevance of the apparent expression between the 2 groups was analyzed using Mann-Whitney U test. P-values $\leq 0.05$ were considered as statistically significant.

Furthermore, the expression profile of each differentially expressed miRNA was used for creation of receiver operating characteristics (ROC) curves, and for estimation of the area under the curve (AUC). This method displays the discriminatory accuracy of the marker for distinguishing between the 2 groups of blood donors.

Using ROC curves the highest Youden index (Y) was calculated. The Youden index is associated with the critical expression point or the optimal threshold value respectively [named cut off-point (COP)] for the biological marker. The COP indicates which value of increased or decreased expression is relevant for the discrimination between malignancy and normal samples and allows assigniment of a particular sample to a certain group.

Based on these COPs the 2 groups were divided into 2 subgroups which exhibited an expression rate over or under the COP. Afterwards, association between altered miRNA expression and malignancy, clinical features and histopathological parameters were calculated using the Chi-square test.

\section{Results}

Demographic characteristics of the study participants. Whole blood samples were collected from 54 OSCC patients (tumor-group), 33 healthy volunteers (control group), 21 patients who relapsed after primary treatment within 1 year, and from 21 patients who did not suffer from a recurrence for at least 1 year after primary treatment. The demographical characteristics of all study participants are summarized in Table I. None of the healthy volunteers had marked oral mucosal pathologies, such as inflammation, hyperplasia or dysplasia. All groups matched in regards to gender and age. There were no statistically relevant differences determined by the Mann-Whitney $\mathrm{U}$ test. The R-OC and the NR-OC groups matched in regards to gender and age $(\mathrm{P}=0.5 ; \mathrm{P}=0.359)$, respectively.

$R T$-qPCR screening for miRNA expression differences between all groups. A significantly different expression of all assessed miRNAs could be ascertained between the OSCC and control group $\left(\mathrm{p}_{\text {miR-186 }}=0.012 ; \mathrm{p}_{\text {miR-3651 }}=0.001 ; \mathrm{p}_{\text {miR-494 }}=0.007\right)$ and the NR-OC group $\left(\mathrm{p}_{\text {miR-186 }}=0.001 ; \mathrm{p}_{\mathrm{miR}-3651}=0.0001 ; \mathrm{p}_{\mathrm{miR}-494}=0.039\right)$, respectively. In contrast there was no significant difference in the expression values between the OSCC and the R-OC group $\left(\mathrm{p}_{\mathrm{miR}-186}=0.94 ; \mathrm{p}_{\mathrm{miR}-3651}=0.89 ; \mathrm{p}_{\mathrm{miR}-494}=0.21\right)$. The comparison of the expression values between the control and the NR-OC group revealed no statistical relevant discrepancy $\left(\mathrm{p}_{\mathrm{miR}}\right.$ ${ }_{186}=0.07 ; \mathrm{p}_{\mathrm{miR}-3651}=0.41 ; \mathrm{p}_{\mathrm{miR}-494}=0.45$ ), while the expression of the miRNAs significantly varied when the control group was compared to the patients who developed recurrence (R-OC group) ( $\left.\mathrm{p}_{\text {miR-186}}=0.03 ; \mathrm{p}_{\mathrm{miR}-3651}=0.0001 ; \mathrm{p}_{\mathrm{miR}-494}=0.003\right)$. A statistically significant difference in expression rate could also be assigned when the recurrence group (R-OC) was compared to the patients who did not develop a recurrence (NR-OC group) within 1 year $\left(\mathrm{p}_{\mathrm{miR}-186}=0.03 ; \mathrm{p}_{\mathrm{miR}-3651}=0.001 ; \mathrm{p}_{\mathrm{miR}-494}=0.003\right)$. The results of the statistical evaluation are summarized in Table III.

$R T$-qPCR screening for miRNA expression between the $R-O C$ and the NR-OC group. The impact of the differences in the expression levels of the miRNAs for the discrimination between the NR-OC and the R-OC groups was more accurately evaluated statistically in order to assess their usefulness for the detection of recurrence and the clinical monitoring of the disease. A significantly different expression could be determined between the NR-OC and the R-OC group for miR-186, miR-3651, and miR-494 (Table IV; Fig. 1). The results are graphically plotted as Box-Whisker plots. Higher $\triangle \mathrm{CT}$ values signify lower miRNA expression levels. RQ values indicate the changes in the expression levels (Table IV; Fig. 1).

miR-186 was significantly decreased in whole blood when recurrence occurred $(\mathrm{P}=0.003)$. It was downregulated 2.1-fold. The expression of the two other miRNAs was significantly increased. The fold-change for miR-3651 was 2-fold whereas this value amounted to 2.3 for miR-494. The increased expression was significantly associated to recurrence $\left(\mathrm{p}_{\mathrm{miR}-3651}=0.001\right.$; $\left.\mathrm{p}_{\mathrm{miR}-494}=0.003\right)$. The results are presented in Fig. 1 and Table IV.

Between the R-OC and the NR-OC group an ROC curve was established and the AUC was determined. All markers yielded a significant AUC value. The upregulated miRNAs, miR-3651 and miR-494 yielded an AUC of 0.80 and 0.78, respectively. The AUC value of the decreased miR-186 amounted to 0.76 (Fig. 2; Table IV). 
Table III. Statistical evaluation of the comparison between all different [test group (OSCC patients), control (healthy volunteers) and follow-up] groups.

P-values ${ }^{\mathrm{a}}$

Compared groups hsa-miR-186-5p hsa-miR-3651 hsa-miR-494-5p

Test group vs. control group

Test group vs. R-OC group

Test group vs. NR-OC group

Control group vs. R-OC group

Control group vs. NR-OC group

R-OC group vs. NR-OC group

0.012
0.94
0.001
0.03
0.07
0.003

0.012

0.001

0.89

0.0001

0.0001

0.41

0.001
0.007

0.21

0.039

0.003

0.451

0.003

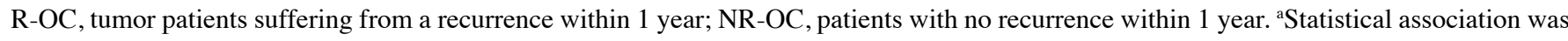
determined using Mann-Whitney-U test.
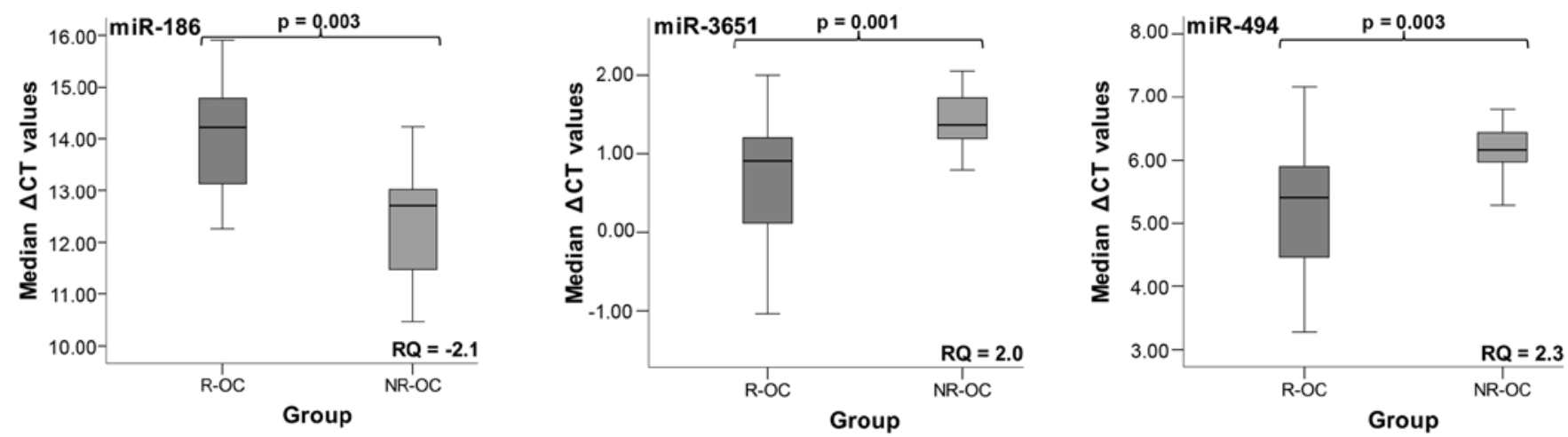

Figure 1. Box-Whisker plots of median expression rates of the miRNAs in whole blood of the R-OC and NR-OC groups derived from RT-qPCR. The expression values are expressed as the median $\triangle \mathrm{CT}$ values. Higher $\triangle \mathrm{CT}$ values signify decreased miRNA expression. The median, the interquartile range and the standard deviation are presented. The fold-change of miRNA expression (RQ) was carried out by the $\Delta \Delta \mathrm{CT}$ method comparing the average $\Delta \mathrm{CT}$ values of the 2 groups [formula: $2^{-(\Delta \mathrm{CT} R-\mathrm{OC}-\Delta \mathrm{CT} \mathrm{NR}-\mathrm{OC})}$. The expression was statistically relevant and altered when comparing the R-OC group to the NR-OC group. Statistical evaluation was carried out using the Mann-Whitney U test.

Table IV. Statistical evaluation of the comparison of the R-OC (tumor patients suffering from recurrence within 1 year) and the NR-OC group (recurrence-free patients) based on the determined $\Delta \mathrm{CT}$ values.

\begin{tabular}{lrcccccc}
\hline miRNA & FC & AUC & Y & COP $(\Delta C T)$ & P-value $^{a}$ & Sensitivity $(\%)$ & Specificity (\%) \\
\hline hsa-miR-186-5p & -2.1 & 0.76 & 0.524 & 13.39 & 0.003 & 71,4 & 81 \\
hsa-miR-3651 & 2.0 & 0.80 & 0.525 & 1.16 & 0.001 & 81 & 71,4 \\
hsa-miR-494-5p & 2.3 & 0.78 & 0.476 & 5.87 & 0.003 & 71,4 & 76,2 \\
\hline
\end{tabular}

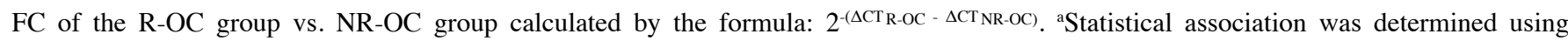
Mann-Whitney-U test. FC, fold change; AUC, area under the curve; Y, Youden index; COP, cut-off point given as $\Delta \mathrm{CT}$ value.

The highest Youden indices were 0.476 for miR-494, 0.525 for miR-3651 and 0.524 for miR-186 (Table IV). The optimal threshold values/cut-off points (COP) expressed as a $\triangle \mathrm{CT}$ value were 5.87 for miR-494, 1.16 for miR-3651 and 13.39 for miR-186. For the miRNAs miR-494 and miR-3651 a $\Delta \mathrm{CT}$ under the COP (signifying upregulation) was considered to be positive for the recurrence corresponding to an increased abundance of the marker in whole blood. For miR-186 a $\Delta \mathrm{CT}$ value over the COP (signifying downregulation) was positive for recurrence. Using the determined COPs the 2 groups were divided into positive and negative specimens. The changes in the expression rates of the miRNAs were statistically relevant and associated to recurrence. The results are diagrammed in Fig. 3 and summarized in Table V.

Out of the patients with recurrence $71.4 \%$ (15/21) exhibited decreased levels of miR-186 and 71.4\% (15/21) and 

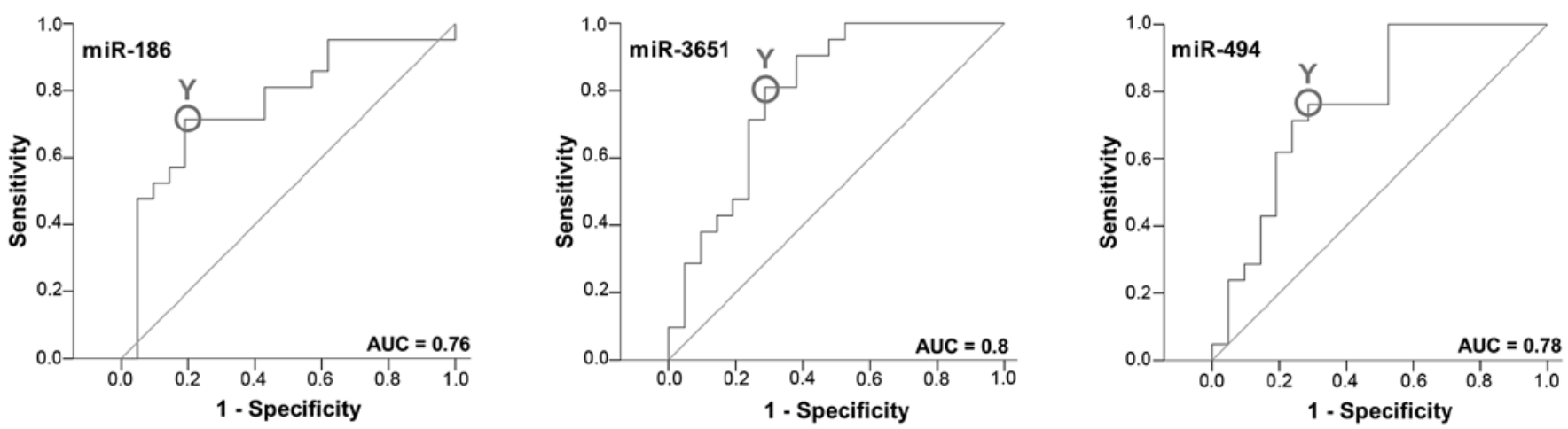

Figure 2. ROC curves for miRNAs based on the RT-qPCR data derived from whole blood of the R-OC and NR-OC groups. The diagrams plot the sensitivity (true positive rate) vs. 1 - specificity (false-positive rate) over all possible $\triangle \mathrm{CT}$ values. The AUC values indicate that the 2 groups could be distinguished by expression analysis of the markers. The circle shows the point of highest Youden $(\mathrm{Y})$ indices which are associated with the COP. The determined values of AUC, Y and COP for the examined miRNAs are listed in Table IV. miRNA, microRNA; RT-qPCR, quantitative RT-PCR; AUC, area under the curve; COP, cut-off point.

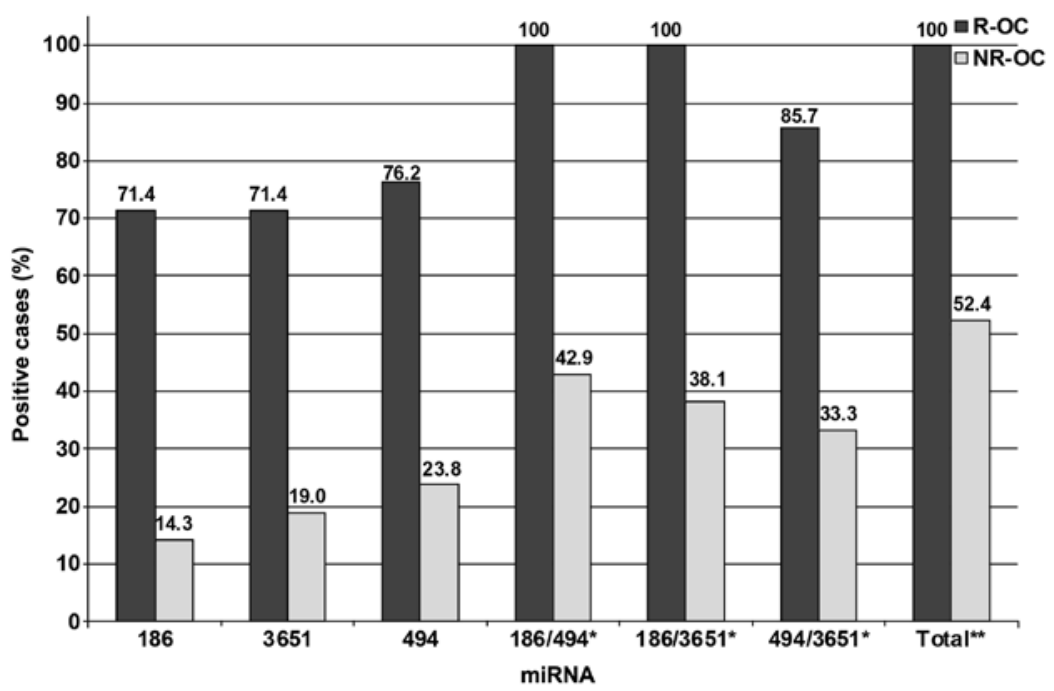

Figure 3. Division of the R-OC and NR-OC groups into positive and negative subgroups based on ascertained COPs of single miRNAs given as $\Delta C T$ values. The specimens were considered positive (recurrent) if the values lay below (for miR-494 and miR-3651) or above (miR-186) the COP. "Total positive cases with the combination of 2 miRNAs. ${ }^{* *}$ Positive for at least 1 of the examined miRNAs. Altered expression levels in blood between the 2 groups appeared to be significant.

$76.2 \%(16 / 21)$ exhibited increased values of miR-3651 and miR-494, respectively. Only $14.3 \%$ (3/21) of the whole blood samples from the NR-OC group exhibited decreased levels of miR-186. Increased levels in blood of patients suffering from NR-OC were revealed in $19.0 \%$ (4/21) of the cases for miR-3651 and in $23.8 \%$ (5/21) for miR-494, respectively. In order to ascertain the utility of the combination of 2 miRNAs as prognostic tools 3 additional arrangements of the investigated miRNAs were made. When a combination of miR-186/miR-494 and miR-186/miR-3651 was assessed $100 \%$ of the R-OC specimens exhibited positivity for altered expression, whereas only 42.9 and $38.1 \%$ of the NR-OC samples showed positivity. When, using the combination miR-494/miR-3651 $85.7 \%$ of the patients with recurrence were positive, whereas only $33.3 \%$ of the patients with no recurrence exhibited specific altered expression of 1 of these 2 markers. The correlation between recurrence and the detection of altered expression rates was significant for all the investigated miRNAs and their various combinations $(\mathrm{P}<0.01)$. Moreover, $100 \%$ of the blood samples of patients suffering from recurrence exhibited altered expression of at least 1 of the examined miRNAs whereas in only $52.4 \%$ of the disease-free patients such an altered abundance was evident. Thus, the association to recurrence was statistically relevant $(\mathrm{P}=0.0001)$.

The sensitivity of miRNAs, miR-186, miR-3651 and miR-494 amounted to $0.714,0.714$ and 0.762 , respectively. The values of the specificity of miR-186, miR-3651 and miR-494 were $0.857,0.81$ and 0.762 . The analyzed combinations reached different values for sensitivity: miR-186/miR-494, 1; miR-186/miR-3651, 1 and miR-494/miR-3651,0.857; and specificity: miR-186/miR-494, 0.571; miR-186/miR-3651, 0.619 and $\mathrm{miR}-494 / \mathrm{miR}-3651,0.667$.

\section{Discussion}

Approximately $50 \%$ of patients with OSCC present with metastatic disease or local recurrence at the time of initial diagnosis or in the aftercare, leading to an unfavorable 
Table V. Association between altered expression rates of each individual miRNA and recurrence.

\begin{tabular}{|c|c|c|c|c|c|c|}
\hline \multirow[b]{2}{*}{ miRNA } & \multicolumn{2}{|c|}{ Positive $\mathrm{n} / \%$} & \multirow[b]{2}{*}{ P-value } & \multirow[b]{2}{*}{ Sensitivity } & \multirow[b]{2}{*}{ Specificity } & \multirow{2}{*}{$\begin{array}{l}\text { Predictive value } \\
\text { (Positive/negative) }\end{array}$} \\
\hline & $\mathrm{R}-\mathrm{OC}$ & NR-OC & & & & \\
\hline $186-5 p$ & $15 / 71.4$ & $3 / 14.3$ & 0.001 & 0.714 & 0.857 & $0.83 / 0.75$ \\
\hline 3651 & $15 / 71.4$ & $4 / 19.0$ & 0.001 & 0.714 & 0.81 & $0.79 / 0.74$ \\
\hline $494-5 p$ & $16 / 76.2$ & $5 / 23.8$ & 0.002 & 0.762 & 0.762 & $0.762 / 0.76$ \\
\hline $186 / 494^{a}$ & $21 / 100$ & $9 / 42.9$ & 0.0001 & 1 & 0.571 & $0.7 / 1$ \\
\hline $186 / 3651^{\mathrm{a}}$ & $21 / 100$ & $8 / 38.1$ & 0.0001 & 1 & 0.619 & $0.81 / 1$ \\
\hline $494 / 3651^{\mathrm{a}}$ & $18 / 85.7$ & $7 / 33.3$ & 0.001 & 0.857 & 0.667 & $0.72 / 0.82$ \\
\hline Total $^{\mathrm{b}}$ & $21 / 100$ & $11 / 52.4$ & 0.0001 & 1 & 0.476 & $0.656 / 1$ \\
\hline
\end{tabular}

Positivity of the blood samples of the particular patients was ascertained using the determined COP of each respective miRNA. R-OC $(\mathrm{n}=21)$, OSCC patients suffering from a recurrence within 1 year; NR-OC $(n=21)$, patient without recurrence within 1 year. Value of statistical relevance

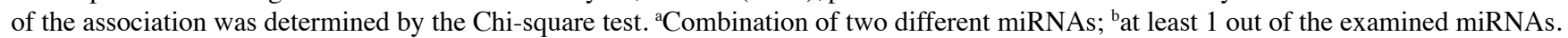

prognosis (38). Therefore, early detection of primary and/or recurrent OSCC by routine laboratory tests is required $(4,5,7)$. Moreover, the monitoring of the response to successful therapy by serial assessment of biomarkers, showing different pretreatment and post-treatment levels is much-warranted (39). Tumor biomarkers in blood and saliva may allow an earlier detection of the primary and/or recurrent disease, and may serve as possible predictors of prognosis for OSCC. Unfortunately, most of the identified markers lack the required specificity and sensitivity. Therefore, the importance of developing useful diagnostic and monitoring tools is emphasized in order to increase initial recognition of patients with a higher risk of recurrent disease and to improve long-term clinical outcomes by allowing for a more aggressive treatment approach and by carrying out a closer follow-up of these patients in the aftercare (8).

miRNAs are important regulatory molecules and are shown to be involved in disease pathogenesis. The perceived opportunity for their use as clinical markers has loomed particularly large in neoplastic disease, where alterations in cancer cells are thought to be reflected in the extracellular space as affected cells release upregulated miRNAs or fail to release apparently downregulated species (18). Additionally, miRNAs are very stable and can therefore be detected in several body fluids including serum, plasma, saliva and whole blood. Several studies have demonstrated the usefulness of circulating miRNAs as potential biomarkers that may aid in risk assessment, diagnosis, prognosis, and monitoring of disease and of treatment response for OSCC (25-27,33,34,40-47).

In the present study the hypothesis that differential expression of defined miRNAs in whole blood samples of OSCC patients could be associated with recurrence of OSCC and can therefore predict recurrent disease and provide clinically relevant prognostic information for OSCC patients in a postoperative setting was assessed.

In previous studies, we identified 3 differentially expressed miRNAs in whole blood of OSCC patients when compared to that of healthy volunteers $(31,32)$. The miR-494 and miR-3651 concentrations in OSCC patients were increased, whereas miR-186 exhibited decreased abundance. The altered expression rates of these miRNAs were significantly associated to diagnosis. Additionally, overexpression of miR-3651 was significantly correlated to lymph node metastasis, clinical stage and to more dedifferentiated tumors. Thus, it was postulated that these miRNAs may be useful in prognostic applications and may serve as the basis for establishing a minimally invasive method for the detection and monitoring of OSCC $(31,32)$.

In the present study, we confirmed the significant changes in the expression of the investigated miRNAs in OSCC patients compared to healthy controls. The diagnosis was significantly correlated to the detection of altered expression rates of all examined miRNAs. In addition, the changes in miRNA concentrations were significantly different between healthy people and patients with recurrence (R-OC), as well as in OSCC patients compared to patients with no recurrence (NR-OC), respectively. In contrast, these miRNAs were not differentially expressed in whole blood of volunteers when compared to patients with no recurrence or in whole blood of patients suffering from a primary OSCC compared to test subjects who relapsed. These results strengthen the hypothesis that the occurrence of primary as well as of recurrent malignancies can be diagnosed by the expression analysis of these miRNAs in whole blood.

It has already been demonstrated that increased postsurgical concentrations of different miRNAs in plasma, serum and saliva samples of OSCC patients were associated with recurrence and predicted worse clinical outcome (46-49). In the present study, we revealed a significantly different expression of miR-186, miR-3651 and miR-494 in whole blood between the group of patients with recurrence and those with no recurrence that allows for the discrimination between the 2 groups. Moreover, taking into account the COPs the presence of upregulated miR-494 and miR-3651 and downregulated miR-186 in individual pro-bands was significantly correlated with the absence and presence of the recurrence of the disease. Moreover, the markers showed high sensitivity and specificity, and promising positive or negative predictive values. Thus, it appears that these 3 miRNAs are relevant biomarkers in the differentiation of patients with recurrence 
to those with no recurrence. Thus, they could be useful for the establishment of a minimally invasive method based on blood assessment that may provide an early indication of the existence of persistent or recurrent OSCC in an individual patient. Furthermore, it was also revealed that all patients with recurrence were positive for at least 1 differentially expressed miRNA. Thus the sensitivity of this multimarker combination was excellent. However, $52.4 \%$ of the patients with no recurrence were determined as positive. This led to a low degree of specificity. Additionally, the paired combination of the 2 markers resulted in a higher sensitivity of the test, but in contrast in a concomitant loss of specificity. Therefore, in the future it may be useful to explore more than 1 biomarker in order to enhance the diagnostic accuracy $(8,50)$. However, by establishing combinations of markers both the sensitivity and specificity of the test may be investigated and the ratio between these 2 values may be taken into consideration in order to achieve methods with optimal sensitivity as well as the highest possible specificity.

Besides the detection of recurrent disease, many issues could be addressed toward clinical application. For several tumors including OSCC numerous potential miRNAs as valuable biomarkers in predicting the behavior of individual cancers and monitoring therapeutic responses could be displayed. Studies on various tumors evaluating blood miRNAs as biomarkers for OSCC management have been carried out (27,34,43-46,51,52). Moreover, these identified markers may also be applied in the risk assessment of precancerous lesions (53). These studies led to the formulation of the phrase 'liquid biopsy' that may allow for the early warning of oncogenesis, relapse and treatment failure (18). Consequently, our results may encourage the expansion of studies on the evaluation of blood miRNAs as biomarkers for OSCC management and monitoring of the disease. However, further validation in a larger cohort is warranted to fully assess the utility of particular miRNAs as OSCC biomarkers. Additionally, further investigations are warranted for the screening of other miRNAs for clinical use which have already been shown to be valuable tools for these clinical applications. Moreover, their impact in clinical monitoring and prognosis has to be evaluated by particular prospective follow-up studies. Finally in order to translate the promising results into clinical practice these markers have to be validated in well-designed and sufficiently powered multi-centre studies.

In conclusion, altered levels of miR-494, miR-3651 and miR-186 which are differentially expressed in primary OSCC patients were also observed upon disease relapse. Thus, the present study clearly demonstrated the usefulness of circulating miRNAs in the screening of OSCC patients and for the early detection of recurrent and persistent disease. This may provide us with the possibility of setting up a minimally invasive method for OSCC patient stratification according to the risk of recurrence, clinical monitoring and therapeutic response in order to optimize the treatment of patients and to improve tumor outcome.

\section{Acknowledgements}

The authors would like to thank Mrs. S. Schönherr and Mrs. E. Diebel for their valuable technical support.

\section{References}

1. Jemal A, Bray F, Center MM, Ferlay J, Ward E and Forman D: Global cancer statistics. CA Cancer J Clin 61: 69-90, 2011.

2. Yanamoto S, Yamada S, Takahashi H, Yoshitomi I, Kawasaki G, Ikeda H, Minamizato T, Shiraishi T, Fujita S, Ikeda T, et al: Clinicopathological risk factors for local recurrence in oral squamous cell carcinoma. Int J Oral Maxillofac Surg 41: 1195-1200, 2012.

3. Wang B, Zhang S, Yue K and Wang XD: The recurrence and survival of oral squamous cell carcinoma: A report of 275 cases. Chin J Cancer 32: 614-618, 2013.

4. Agra IM, Carvalho AL, Pinto CA, Martins EP, Filho JG, Soares FA and Kowalski LP: Biological markers and prognosis in recurrent oral cancer after salvage surgery. Arch Otolaryngol Head Neck Surg 134: 743-749, 2008.

5. Kalavrezos N and Bhandari R: Current trends and future perspectives in the surgical management of oral cancer. Oral Oncol 46: 429-432, 2010.

6. Ord RA, Kolokythas A and Reynolds MA: Surgical salvage for local and regional recurrence in oral cancer. J Oral Maxillofac Surg 64: 1409-1414, 2006.

7. Schwartz GJ, Mehta RH, Wenig BL, Shaligram C and Portugal LG: Salvage treatment for recurrent squamous cell carcinoma of the oral cavity. Head Neck 22: 34-41, 2000.

8. Søland TM and Brusevold IJ: Prognostic molecular markers in cancer - quo vadis? Histopathology 63: 297-308, 2013.

9. Huang TY, Hsu LP, Wen YH, Huang TT, Chou YF, Lee CF, Yang MC, Chang YK and Chen PR: Predictors of locoregional recurrence in early stage oral cavity cancer with free surgical margins. Oral Oncol 46: 49-55, 2010.

10. Chauhan SS, Kaur J, Kumar M, Matta A, Srivastava G, Alyass A, Assi J, Leong I, MacMillan C, Witterick I, et al: Prediction of recurrence-free survival using a protein expression-based risk classifier for head and neck cancer. Oncogenesis 4: e147, 2015.

11. Komatsu S, Ichikawa D, Hirajima S, Kawaguchi T, Miyamae M, Okajima W, Ohashi T, Arita T, Konishi H, Shiozaki A, et al: Plasma microRNA profiles: Identification of miR-25 as a novel diagnostic and monitoring biomarker in oesophageal squamous cell carcinoma. Br J Cancer 111: 1614-1624, 2014.

12. Lewis BP, Burge CB and Bartel DP: Conserved seed pairing, often flanked by adenosines, indicates that thousands of human genes are microRNA targets. Cell 120: 15-20, 2005.

13. Calin GA and Croce CM: MicroRNA signatures in human cancers. Nat Rev Cancer 6: 857-866, 2006.

14. Lu J, Getz G, Miska EA, Alvarez-Saavedra E, Lamb J, Peck D, Sweet-Cordero A, Ebert BL, Mak RH, Ferrando AA, et al: MicroRNA expression profiles classify human cancers. Nature 435: 834-838, 2005.

15. Cortez MA and Calin GA: MicroRNA identification in plasma and serum: A new tool to diagnose and monitor diseases. Expert Opin Biol Ther 9: 703-711, 2009.

16. Momen-Heravi F, Trachtenberg AJ, Kuo WP and Cheng YS: Genomewide study of salivary microRNAs for detection of oral cancer. J Dent Res 93 (Suppl 7): 86S-93S, 2014.

17. Mitchell PS, Parkin RK, Kroh EM, Fritz BR, Wyman SK, Pogosova-Agadjanyan EL, Peterson A, Noteboom J, O'Briant KC, Allen A, et al: Circulating microRNAs as stable blood-based markers for cancer detection. Proc Natl Acad Sci USA 105: 10513-10518, 2008.

18. Witwer KW: Circulating microRNA biomarker studies: Pitfalls and potential solutions. Clin Chem 61: 56-63, 2015.

19. Cappelletti V, Appierto V, Tiberio P, Fina E, Callari M and Daidone MG: Circulating biomarkers for prediction of treatment response. J Natl Cancer Inst Monogr 2015: 60-63, 2015.

20. Tiberio P, Callari M, Angeloni V, Daidone MG and Appierto V: Challenges in using circulating miRNAs as cancer biomarkers. BioMed Res Int 2015: 731479, 2015.

21. Chen X, Ba Y, Ma L, Cai X, Yin Y, Wang K, Guo J, Zhang Y, Chen J, Guo X, et al: Characterization of microRNAs in serum: A novel class of biomarkers for diagnosis of cancer and other diseases. Cell Res 18: 997-1006, 2008.

22. Allegra A, Alonci A, Campo S, Penna G, Petrungaro A, Gerace D and Musolino C: Circulating microRNAs: New biomarkers in diagnosis, prognosis and treatment of cancer (Review). Int $\mathbf{J}$ Oncol 41: 1897-1912, 2012.

23. Schwarzenbach H, Nishida N, Calin GA and Pantel K: Clinical relevance of circulating cell-free microRNAs in cancer. Nat Rev Clin Oncol 11: 145-156, 2014. 
24. Iorio MV and Croce CM: MicroRNA dysregulation in cancer: Diagnostics, monitoring and therapeutics. A comprehensive review. EMBO Mol Med 4: 143-159, 2012.

25. Gomes CC, de Sousa SF and Gomez RS: MicroRNAs: Small molecules with a potentially role in oral squamous cell carcinoma. Curr Pharm Des 19: 1285-1291, 2013.

26. Wu BH, Xiong XP, Jia J and Zhang WF: MicroRNAs: New actors in the oral cancer scene. Oral Oncol 47: 314-319, 2011.

27. Soga D, Yoshiba S, Shiogama S, Miyazaki H, Kondo S and Shintani S: microRNA expression profiles in oral squamous cell carcinoma. Oncol Rep 30: 579-583, 2013.

28. Zahran F, Ghalwash D, Shaker O, Al-Johani K and Scully C: Salivary microRNAs in oral cancer. Oral Dis 21: 739-747, 2015.

29. Hanash SM, Baik CS and Kallioniemi O: Emerging molecular biomarkers - blood-based strategies to detect and monitor cancer. Nat Rev Clin Oncol 8: 142-150, 2011.

30. Wu JY, Yi C, Chung HR, Wang DJ, Chang WC, Lee SY, Lin CT, Yang YC and Yang WC: Potential biomarkers in saliva for oral squamous cell carcinoma. Oral Oncol 46: 226-231, 2010.

31. Ries J, Vairaktaris E, Agaimy A, Kintopp R, Baran C, Neukam FW and Nkenke E: miR-186, miR-3651 and miR-494: Potential biomarkers for oral squamous cell carcinoma extracted from whole blood. Oncol Rep 31: 1429-1436, 2014.

32. Ries J, Vairaktaris E, Kintopp R, Baran C, Neukam FW and Nkenke E: Alterations in miRNA expression patterns in whole blood of OSCC patients. In Vivo 28: 851-861, 2014.

33. Masood Y, Kqueen CY and Rajadurai P: Role of miRNA in head and neck squamous cell carcinoma. Expert Rev Anticancer Ther 15: 183-197, 2015.

34. Ganci F, Sacconi A, Manciocco V, Sperduti I, Battaglia P, Covello R, Muti P, Strano S, Spriano G, Fontemaggi G, et al: MicroRNA expression as predictor of local recurrence risk in oral squamous cell carcinoma. Head Neck 38 (Suppl 1): E189-E197, 2016.

35. Xiao W, Bao ZX, Zhang CY, Zhang XY, Shi LJ, Zhou ZT and Jiang WW: Upregulation of miR-31* is negatively associated with recurrent/newly formed oral leukoplakia. PLoS One 7: e38648, 2012.

36. Gorenchtein M, Poh CF, Saini R and Garnis C: MicroRNAs in an oral cancer context - from basic biology to clinical utility. J Dent Res 91: 440-446, 2012.

37. Livak KJ and Schmittgen TD: Analysis of relative gene expression data using real-time quantitative PCR and the $2^{-\Delta \Delta C_{\mathrm{T}}}$ method. Methods 25: 402-408, 2001.

38. Kowalski LP and Sanabria A: Elective neck dissection in oral carcinoma: A critical review of the evidence. Acta Otorhinolaryngol Ital 27: 113-117, 2007.

39. Grimm M, Kraut W, Hoefert S, Krimmel M, Biegner T, Teriete P, Cetindis M, Polligkeit J, Kluba S, Munz A, et al: Evaluation of a biomarker based blood test for monitoring surgical resection of oral squamous cell carcinomas. Clin Oral Investig 20: 329-338, 2016.
40. Gomes CC and Gomez RS: MicroRNA and oral cancer: Future perspectives. Oral Oncol 44: 910-914, 2008

41. Yoshizawa JM and Wong DT: Salivary microRNAs and oral cancer detection. Methods Mol Biol 936: 313-324, 2013.

42. Zen K and Zhang CY: Circulating microRNAs: A novel class of biomarkers to diagnose and monitor human cancers. Med Res Rev 32: 326-348, 2012.

43. Summerer I, Niyazi M, Unger K, Pitea A, Zangen V, Hess J, Atkinson MJ, Belka C, Moertl S and Zitzelsberger H: Changes in circulating microRNAs after radiochemotherapy in head and neck cancer patients. Radiat Oncol 8: 296, 2013.

44. Gombos K, Horváth R, Szele E, Juhász K, Gocze K, Somlai K, Pajkos G, Ember I and Olasz L: miRNA expression profiles of oral squamous cell carcinomas. Anticancer Res 33: 1511-1517, 2013.

45. Komatsu S, Ichikawa D, Takeshita H, Tsujiura M, Morimura R, Nagata H, Kosuga T, Iitaka D, Konishi H, Shiozaki A, et al: Circulating microRNAs in plasma of patients with oesophageal squamous cell carcinoma. Br J Cancer 105: 104-111, 2011.

46. Liu CJ, Kao SY, Tu HF, Tsai MM, Chang KW and Lin SC: Increase of microRNA $m i R-31$ level in plasma could be a potential marker of oral cancer. Oral Dis 16: 360-364, 2010.

47. Lin SC, Liu CJ, Lin JA, Chiang WF, Hung PS and Chang KW: $m i R-24$ up-regulation in oral carcinoma: Positive association from clinical and in vitro analysis. Oral Oncol 46: 204-208, 2010

48. Wong TS, Liu XB, Wong BY, Ng RW, Yuen AP and Wei WI: Mature miR-184 as potential oncogenic microRNA of squamous cell carcinoma of tongue. Clin Cancer Res 14: 2588-2592, 2008.

49. Liu CJ, Lin SC, Yang CC, Cheng HW and Chang KW: Exploiting salivary $m i R-31$ as a clinical biomarker of oral squamous cell carcinoma. Head Neck 34: 219-224, 2012.

50. Grimm M, Schmitt S, Teriete P, Biegner T, Stenzl A, Hennenlotter J, Muhs HJ, Munz A, Nadtotschi T, König K, et al: A biomarker based detection and characterization of carcinomas exploiting two fundamental biophysical mechanisms in mammalian cells. BMC Cancer 13: 569, 2013.

51. Friedman EB, Shang S, de Miera EV, Fog JU, Teilum MW, Ma MW, Berman RS, Shapiro RL, Pavlick AC, Hernando E, et al: Serum microRNAs as biomarkers for recurrence in melanoma. $\mathrm{J}$ Transl Med 10: 155, 2012.

52. Schwarzenbach H, Hoon DS and Pantel K: Cell-free nucleic acids as biomarkers in cancer patients. Nat Rev Cancer 11: 426-437, 2011.

53. Maclellan SA, Lawson J, Baik J, Guillaud M, Poh CF and Garnis C: Differential expression of miRNAs in the serum of patients with high-risk oral lesions. Cancer Med 1: 268-274, 2012. 